\title{
LINEAR SUPERPOSITIONS IN NONLINEAR WAVE EQUATIONS
}

\author{
A. S. Fokas and Q. M. Liu \\ Dedicated to Martin D. Kruskal on the occasion of his 70th birthday.
}

There exist nonlinear wave equations which admit a linear superposition of their traveling wave solutions. An example of such an equation is the generalized Boussinesq equation

$$
u_{t t}+u_{x x}-u_{x x x x}+\left(u_{x} u_{t}\right)_{x}=0
$$

which possesses the exact solution

$$
\begin{aligned}
u(x, t)= & -3 \frac{\sqrt{1+k^{2}}}{k} \tanh \left[\frac{1}{2} \sqrt{1+k^{2}}\left(x+k t+\delta_{1}\right)\right] \\
& +3 \frac{\sqrt{1+k^{2}}}{k} \tanh \left[\frac{1}{2} \sqrt{1+k^{2}}\left(x-k t+\delta_{2}\right)\right]
\end{aligned}
$$

where $k, \delta_{1}, \delta_{2}$ are arbitrary constants. This solution was obtained in [1], where it also was shown that the solution corresponds to a non-classical symmetry. It is interesting that the solution (2) looks like the elastic interaction of two "soliton" solutions, although it appears that (1) is not an integrable equation.

In this note: (a) It is shown that such exact solutions appear in a large class of nonlinear equations, and that these solutions correspond to linear generalized conditional symmetries (GCS). Particular classes of such equations are

$$
L_{1}\left(\partial_{x}, \partial_{t}\right) u=L_{2}\left(\partial_{x}, \partial_{t}\right)\left(u_{x} u_{t}\right)
$$

and

$$
L\left(\partial_{x}, \partial_{t}\right) u=f_{1}\left(u_{t}+k u_{x}\right)+f_{2}\left(u_{t}-k u_{x}\right), \quad k \text { constant }
$$

where $L_{1}, L_{2}, L$ are arbitrary linear differential operators of $\partial_{x}$ and $\partial_{t}$, and $f_{1}, f_{2}$ are arbitrary differentiable functions of the arguments indicated. (Equation (1) is a particular case of (3) where $L_{1}=\partial_{t}^{2}+\partial_{x}^{2}-\partial_{x}^{4}$ and $L_{2}=-\partial_{x}$.)

(b) The general form of all nonlinear evolution equations admitting a given linear GCS is explicitly given. A particular case of this construction is that the most general evolution equation of degree $\leq 3$ admitting the linear GCS $\sigma=u_{x x x}+u_{x}$ is

$$
u_{t}=u_{x x} F_{1}\left(u_{x x}^{2}+u_{x}^{2}, u_{x x}+u\right)+u_{x} F_{2}\left(u_{x x}^{2}+u_{x}^{2}, u_{x x}+u\right)+F_{3}\left(u_{x x}^{2}+u_{x}^{2}, u_{x x}+u\right)
$$

Received October 4, 1995, revised November 14, 1996.

1991 Mathematics Subject Classification: 35Q53, 35C05.

Key words and phrases: nonlinear wave equations, linear superposition, generalized conditional symmetries. 
where $F_{1}, F_{2}, F_{3}$ are arbitrary differentiable functions of the arguments indicated. If $F_{1}(r, s)=1-2 s, F_{2}=0, F_{3}(r, s)=r+s^{2}$, equation (5) becomes the physically interesting equation

$$
u_{t}=u_{x x}+u_{x}^{2}+u^{2}
$$

(see [2]).

Notation. $A(u)$ will denote a differentiable function of $u$ and of all derivatives of $u$ with respect to $x$ and to $t$, i.e.,

$$
A(u)=A\left(u, u_{x}, u_{t}, u_{x x}, u_{t t}, u_{x t}, \ldots\right) .
$$

Similarly,

$$
A(u ; v)=A\left(u, u_{x}, u_{t}, u_{x x}, u_{t t}, u_{x t}, \ldots ; v, v_{x}, v_{t}, v_{x x}, v_{t t}, v_{x t}, \ldots\right) .
$$

Prime will denote the Fréchet derivative, i.e.,

$$
K^{\prime}(u)=\frac{\partial K}{\partial u}+\frac{\partial K}{\partial u_{x}} \partial_{x}+\frac{\partial K}{\partial u_{t}} \partial_{t}+\frac{\partial K}{\partial u_{x x}} \partial_{x}^{2}+\frac{\partial K}{\partial u_{t t}} \partial_{t}^{2}+\frac{\partial K}{\partial u_{x} \partial u_{t}} \partial_{x} \partial_{t}+\cdots
$$

The notation $\left.A\right|_{K=0}$ means that the equation $K=0$ and all its differential consequences can be used to compute $A$.

Before deriving the main results, we recall the definition of a GCS. This concept was introduced in [3]. The concept of conditional symmetry was introduced in [4] under the name of non-classical symmetry. The concept of a GCS is a generalization of the concept of a conditional symmetry, in the same way that the concept of a generalized symmetry [5] is a generalization of the concept of a symmetry.

Definition. [3] $\sigma(u)$ is a GCS of the equation $K(u)=0$, iff

$$
\left.K^{\prime} \sigma\right|_{K=0}=F(u ; \sigma), \quad F(u ; 0)=0 .
$$

Remark 1. If $F(u ; \sigma)=0$, then (9) becomes the definition of a generalized symmetry $\sigma$ for the equation $K=0$.

Remark 2. Starting from a given PDE, there exists a well-developed algorithm for finding both symmetries and generalized symmetries. This algorithm reduces the problem of finding symmetries to the problem of solving an overdetermined system of linear equations. In contrast, the question of finding conditional symmetries and GCSs is much harder. Indeed, in this case one needs to solve a system of nonlinear equations. For this reason, the authors have suggested an alternative simple approach: Given a GCS of a certain type, find all nonlinear PDE's which admit this GCS. In what follows, we search for equations which admit linear GCS's with constant coefficients.

Proposition 1. Let $L\left(\partial_{x}, \partial_{t}\right)$ and $M\left(\partial_{x}, \partial_{t}\right)$ denote linear differential operators of $\partial_{x}$ and of $\partial_{t}$. The nonlinear equation

$$
L\left(\partial_{x}, \partial_{t}\right) u-N(u)=0
$$

admits the linear GCS

$$
\sigma(u)=M\left(\partial_{x}, \partial_{t}\right) u
$$

if

$$
M N(u)-N^{\prime}(u) M u=F(u ; \sigma), \quad F(u ; 0)=0 .
$$


Proof. $\sigma$ is a GCS of (10) iff

$$
\left.\left(L \sigma-N^{\prime} \sigma\right)\right|_{L u=N(u)}=F(u ; \sigma), \quad F(u ; 0)=0,
$$

or

$$
\left.\left(L M u-N^{\prime} M u\right)\right|_{L u=N(u)}=F(u ; \sigma), \quad F(u ; 0)=0,
$$

which becomes (11) by replacing $L u$ with $N(u)$.

Proposition 2. The nonlinear equation (3) admits the linear GCS

$$
\sigma=u_{t t}-k^{2} u_{x x}, \quad k \text { constant. }
$$

This GCS gives rise to the solution

$$
u=f(x+k t)+g(x-k t)
$$

where $f(x)$ and $g(x)$ are the solutions of the following ODEs:

$$
\begin{gathered}
L_{1}\left(\partial_{x}, k \partial_{x}\right) f(x)=k L_{2}\left(\partial_{x}, k \partial_{x}\right)\left(\frac{d f(x)}{d x}\right)^{2}+\lambda, \\
L_{1}\left(\partial_{x},-k \partial_{x}\right) g(x)=-k L_{2}\left(\partial_{x},-k \partial_{x}\right)\left(\frac{d g(x)}{d x}\right)^{2}-\lambda,
\end{gathered}
$$

$\lambda$ a constant.

Proof. Without loss of generality we let $L_{2}=I$. Then equation (3) is a special case of equation (10). We will verify that if

$$
N(u)=u_{x} u_{t} \quad \text { and } \quad M=\partial_{t}^{2}-k^{2} \partial_{x}^{2},
$$

then (11) is satisfied. Indeed, since $N^{\prime} g=u_{t} g_{x}+u_{x} g_{t}$,

$$
\begin{aligned}
M N(u)-N^{\prime} M u & =\left(\partial_{t}^{2}-k^{2} \partial_{x}^{2}\right) u_{x} u_{t}-u_{t}\left(\partial_{t}^{2}-k^{2} \partial_{x}^{2}\right) u_{x}-u_{x}\left(\partial_{t}^{2}-k^{2} \partial_{x}^{2}\right) u_{t} \\
& =2 u_{x t}\left(u_{t t}-k^{2} u_{x x}\right),
\end{aligned}
$$

and (11) is valid with $F(u ; \sigma)=2 u_{x t} \sigma$.

Proposition 3. The nonlinear equation (4) also admits the linear GCS (12). This $G C S$ gives rise to solution (13) where $f(x)$ and $g(x)$ are the solutions of the ODEs

$$
L\left(\partial_{x}, k \partial_{x}\right) f(x)=f_{1}\left(2 k \frac{d f(x)}{d x}\right)+\lambda, \quad L\left(\partial_{x},-k \partial_{x}\right) g(x)=f_{2}\left(-2 k \frac{d g(x)}{d x}\right)-\lambda .
$$

Proof. Let $u_{t}+k u_{x}=U, u_{t}-k u_{x}=V$. Now $N(u)=f_{1}+f_{2}$,

$$
\begin{aligned}
M N(u)-N^{\prime} M u= & \left(\partial_{t}^{2}-k^{2} \partial_{x}^{2}\right)\left(f_{1}+f_{2}\right) \\
& -\left[f_{1 U}\left(\partial_{t}+k \partial_{x}\right)+f_{2 V}\left(\partial_{t}-k \partial_{x}\right)\right]\left(u_{t t}-k^{2} u_{x x}\right) \\
= & {\left[f_{1 U U}\left(u_{t t}+k^{2} u_{x x}+2 k u_{x t}\right)+f_{1 V V}\left(u_{t t}+k^{2} u_{x x}-2 k u_{x t}\right)\right] \sigma . }
\end{aligned}
$$


Examples. In addition to (1), the following are interesting examples of equations of the form (3):

(i) The integrable Boussinesq type equation [6],

$$
u_{x x t}-u_{t}-u_{x}+u_{t} u_{x}=0
$$

which admits the solution [7]

$$
\begin{aligned}
u(x, t)= & 3 \sqrt{\frac{k+1}{k}} \tanh \left[\frac{1}{2} \sqrt{\frac{k+1}{k}}\left(x+k t+\delta_{1}\right)\right] \\
& +3 \sqrt{\frac{k-1}{k}} \tanh \left[\frac{1}{2} \sqrt{\frac{k-1}{k}}\left(x-k t+\delta_{2}\right)\right] .
\end{aligned}
$$

(ii) The following variation of the potential $\mathrm{KdV}$ equation

$$
u_{t}+u_{x}-u_{x x x}-3 u_{x} u_{t}=0
$$

which admits the solution

$$
\begin{aligned}
u(x, t)= & \frac{\sqrt{1+k}}{k} \tanh \left[\frac{1}{2} \sqrt{1+k}\left(x+k t+\delta_{1}\right)\right] \\
& -\frac{\sqrt{1-k}}{k} \tanh \left[\frac{1}{2} \sqrt{1-k}\left(x-k t+\delta_{2}\right)\right] .
\end{aligned}
$$

This equation to our knowledge is not integrable.

(iii) The linearizable Thomas equation [8]

$$
u_{x t}+u_{x}+u_{t}+u_{x} u_{t}=0
$$

which admits the solution

$$
u(x, t)=\partial_{x}\left[\frac{k+1}{C_{1} e^{\frac{k+1}{k}(x+k t)}-k}+\frac{k-1}{C_{2} e^{\frac{k-1}{k}(x-k t)}-k}\right] .
$$

Proposition 4. The evolution equation

$$
u_{t}=K\left(u, u_{x}, \ldots, \partial_{x}^{n-1} u\right), \quad K \text { polynomial },
$$

admits the linear GCS

$$
\sigma(u)=\left[\prod_{i=1}^{n}\left(\partial_{x}+a_{i}\right)\right] u, \quad a_{i} \text { constants, } a_{i} \neq a_{j} \text { for } i \neq j
$$

iff $K$ is a linear combination of the functions

$$
\prod_{i=1}^{n}\left\{\left[\prod_{j \neq i}\left(\partial_{x}+a_{j}\right)\right] u\right\}^{m_{i}}, \quad \sum_{j=1}^{n} m_{j} a_{j}=a_{i} \text { for some } 1 \leq i \leq n .
$$


Proof. We note that when $u$ satisfies $\sigma(u)=0$,

$$
\begin{aligned}
\partial_{x}\left\{\left[\prod_{j \neq i}\left(\partial_{x}+a_{j}\right)\right] u\right\}^{m_{i}}= & m_{i}\left\{\left[\prod_{j \neq i}\left(\partial_{x}+a_{j}\right)\right] u\right\}^{m_{i}-1} \partial_{x}\left\{\left[\prod_{j \neq i}\left(\partial_{x}+a_{j}\right)\right] u\right\} \\
= & m_{i}\left\{\left[\prod_{j \neq i}\left(\partial_{x}+a_{j}\right)\right] u\right\}^{m_{i}-1}\left[\left(\partial_{x}+a_{i}\right)-a_{i}\right] \\
& \times\left\{\left[\prod_{j \neq i}\left(\partial_{x}+a_{j}\right)\right] u\right\} \\
= & -m_{i} a_{i}\left\{\left[\prod_{j \neq i}\left(\partial_{x}+a_{j}\right)\right] u\right\}^{m_{i}} .
\end{aligned}
$$

Therefore

$$
\begin{aligned}
\prod_{k=1}^{n}\left(\partial_{x}\right. & \left.+a_{k}\right) \prod_{i=1}^{n}\left\{\left[\prod_{j \neq i}\left(\partial_{x}+a_{j}\right)\right] u\right\}^{m_{i}} \\
& =\prod_{k=1}^{n}\left(a_{k}-\sum_{j=1}^{n} m_{j} a_{j}\right) \prod_{i=1}^{n}\left\{\left[\prod_{j \neq i}\left(\partial_{x}+a_{j}\right)\right] u\right\}^{m_{i}} .
\end{aligned}
$$

We note that (17) consists of all the equations of the form (15) which admits the GCS (16), since $u, u_{x}, \ldots, \partial_{x}^{n-1} u$ can be expressed by $\left[\prod_{j \neq i}\left(\partial_{x}+a_{j}\right)\right] u$ for $1 \leq i \leq n$.

Remark 3. An equivalent form of $K$ is the following: $K$ is a linear combination of the functions

$$
\left(\partial_{x}^{l} u\right) \prod_{i=1}^{n}\left\{\left[\prod_{j \neq i}\left(\partial_{x}+a_{j}\right)\right] u\right\}^{m_{i}}, \quad 0 \leq l \leq n-1, \quad \sum_{i=1}^{n} m_{i} a_{i}=0 .
$$

The form (18) is illustrated below:

(i) $n=1$. Then $l$ is 0 and (18) yield the function $u$. Thus the most general equation of the form (15) admitting the symmetry $\sigma=u_{x}+a u$ is $u_{t}=c u, a, c$ constants.

(ii) $n=2$. Then $l$ can be 0 and 1 , and (18) yield the functions

$$
u\left(u_{x}+a_{1} u\right)^{m_{1}}\left(u_{x}+a_{2} u\right)^{m_{2}} \text { and } u_{x}\left(u_{x}+a_{1} u\right)^{m_{1}}\left(u_{x}+a_{2} u\right)^{m_{2}}
$$

where $a_{1} m_{1}+a_{2} m_{2}=0$. Thus the most general equation of the form (15) admitting the symmetry

$$
\sigma=u_{x x}+\left(a_{1}+a_{2}\right) u_{x}+a_{1} a_{2} u, \quad a_{1}, a_{2} \text { constants, }
$$

is equation (15) where $K$ is the linear combination of the functions

$$
\begin{array}{ll}
\left(c_{1} u+c_{2} u_{x}\right)\left(u_{x}+a_{1} u\right)^{m_{1}}\left(u_{x}+a_{2} u\right)^{m_{2}}, & c_{1}, c_{2}, m_{1}, m_{2} \text { constants, } \\
a_{1} m_{1}+a_{2} m_{2}=0 .
\end{array}
$$

Remark 4. Some of the solutions discussed here were obtained in [1] and [7] using the usual (as opposed to generalized) conditional symmetries. However, the conditional symmetries used in [1] and [7] involved explicit $x$ and $t$ dependence, while the GCS used here are of constant coefficients. The advantage of working with GCS of constant coefficients is that it is straightforward to find all nonlinear PDE's which admit these 
GCSs (see Proposition 4). In this way the results obtained in [1] and [7] can be extended considerably.

Acknowledgements. This work was partially supported by the National Science Foundation DMS-91116111 and by the Air Force Office of Scientific Research F4962093-1-0088.

\section{References}

1. G. W. Bluman and J. D. Cole, The general similarity solution of the heat equation, J. Math. Mech. 18 (1969), 1025-1042.

2. P. A. Clarkson and E. L. Mansfield, Algorithms for the nonclassical method of symmetry reductions, to appear in SIAM J. Appl. Math.

3.

4. A. S. Fokas and Q. M. Liu, Nonlinear interaction of traveling waves of nonintegrable equations, Phys. Rev. Lett. 72 (1994), 3293-3296.

5. V. A. Galaktionov, On new exact blow-up solutions for nonlinear heat conduction equations with source and applications, Differential Integral Equations 3 (1990), 863-874.

6. I. M. Gel'fand and L. A. Dikii, Asymptotic behaviour of the resolvent of Sturm-Liouville equations and the algebra of the Korteweg-de Vries equations, Uspekhi Mat. Nauk 30 (1975), 67-100.

7. R. Hirota and J. Satsuma, $N$-soliton solutions of model equations for shallow water waves, J. Phys. Soc. Japan 40 (1976), 611-612.

8. H. C. Thomas, Heterogeneous ion exchange in a flowing system, J. Amer. Chem. Soc. 66 (1944), 1664-1666.

(Fokas) Department of Mathematics, Imperial College of Science, Technology and Medicine, LONDON, SW7 2BZ, U.K.

and

Institute for Nonlinear StUdies, Clarkson University, Potsdam, New York 13699-5815, U.S.A.

(Liu) Department of Mathematics and Computer Science, Clarkson University, Potsdam, NEW YORK 13699-5815, U.S.A. 